\title{
Pedagogía para el desarrollo socioemocional. A propósito de la gestión de aprendizaje en el contexto santiaguero
}

\section{Pedagogy to the socio-emotional development. Considering the learn- ing process management in Santiago de Cuba's context}

\section{Osmany Justis Katt}

Dirección de Educación en Santiago de Cuba

\section{Sandra Almestro Rodríguez \\ Olga Silva Astorga}

Escuela Pedagógica Pepito Tey de Santiago de Cuba

\section{Resumen}

Este artículo es resultante investigativa que identifica como problema insuficiencias en la sistematización pedagógica contextualizada de habilidades socioemocionales en la gestión de aprendizaje en la educación santiaguera. Se traza como objetivo la sistematización de la relación habilidades sociales-emociones-gestión de aprendizaje en los procesos pedagógicos. Se emplea una metodología etnográfica que aplicó como técnicas: entrevistas y observaciones participantes semiestructuradas y la comparación multisituada. Los resultados más notorios están en fundamentar una pedagogía social que sitúa al hombre como centro de la formación y a la sociedad como escenario de su existencia presente y futura. Se sistematiza un procedimiento analítico matricial para la implementación de una pedagogía del desarrollo socioemocional en la gestión de aprendizaje.

Palabras clave: etnografía; modelo percibido; modelo operatorio; habilidades socioemocionales y matriz.

\begin{abstract}
This article was conformed as the result of a research that identifies as problem the deficiencies in contextualized pedagogical systematization of socio-emotional skills in the learning process manage went in Santiago's education system. It stated as a goal the systematization of the relation between social-emotional-management learning skills in the pedagogical processes. An ethnographic Methodology is used that applied techniques such as: interviews, semi-structured participative observation and multiplied comparison. The most notorious results are set in the argumentation of a social pedagogy that placed the men as a center of the formation process, and the society as the setting of its future and present existence. A matricidal analytic procedure is systematized to the implementation of the Pedagogy of the social-emotional development in the management learning process.

Keywords: teacher's thinking ethnography; noticed model; operational model; socioemotional skills; pedagogical analysis matrix.
\end{abstract}




\section{INTRODUCCIÓN. Dialéctica entre requisitos y premisas en la gestión de una pedagogía socioemocional.}

Alcanzar correspondencia en el proceso pedagógico entre el empleo de las emociones y las habilidades sociales es requisito para la formación de la personalidad. Docentes y estudiantes deben lograr una relación vincular permeada de sentimiento y emociones en la formación de los conocimientos necesarios para la convivencia en sociedad. Para ello, es necesario fundamentar una pedagogía social que sitúe al hombre como centro de la formación y a la sociedad como escenario de su existencia presente y futura.

La complejidad de la vida en sociedad, la existencia de acentuadas diferencias sociales, de relaciones de pobreza, exclusión, discriminación en la actual época de la globalización y la digitalización imponen a los diferentes contextos de formación estructuras culturales que deben ser estudiadas con posiciones pedagógicas críticas y comprometidas con el futuro de la humanidad. La construcción y circulación de símbolos culturales por los medios de comunicación al nivel mundial ha ido creando una representación global de la vida en sociedad, en ocasiones descontextualizada de las realidades económicas y culturales de muchos grupos sociales, también ha ido generando sobre esta base premisas para la estructuración de los procesos de gestión de aprendizaje por estudiantes y docentes.

La dialéctica que se genera entre requisitos y premisas es objeto de atención en este trabajo que parte de considerar como problema insuficiencias en la sistematización pedagógica contextualizada de las habilidades socioemocionales en la gestión de aprendizaje en la educación santiaguera.

El trabajo es parte de los resultados del proyecto de investigación y desarrollo: Profesionalización de los Docentes en Gestión de Aprendizaje en el Contexto Santiaguero (PRODOGESANTIAGO), que se realiza en la provincia de Santiago de Cuba. Persigue como objetivo la sistematización teórica de la relación habilidades sociales-emociones-gestión de aprendizaje en los procesos pedagógicos en las instituciones escolares. 


\section{METOdOLOGÍA EMPLEADA PARA LA SISTEMATIZACIÓN DE LA RELACIÓN LA RELACIÓN HABILIDADES SOCIALES-EMOCIONES- GESTIÓN DE APRENDIZAJE}

Se utilizó la etnografía para realizar el trabajo de campo a través de diferentes técnicas como entrevista y observación participantes semiestructuradas y la comparación multisituada apoyados en el procedimiento dialéctico descriptivo del modelo cultural percibido y el modelo cultural operatorio sistematizado por Justis (2013). Ello permitió obtener variedad y suficiencia de información para establecer un estado de la cuestión. Con la realización de grupos de discusión y focales se valoró el impacto de las actividades realizadas y se generaron soluciones que perfeccionaron las maneras de hacer en la gestión de aprendizaje en el contexto santiaguero.

Se siguió una estrategia metodológica general que realiza un estudio etnográfico para la determinación de los modelos culturales percibidos y se complementó con un estudio bibliográfico para contrastarlo con los modelos culturales operatorios de las ciencias pedagógicas.

La muestra seleccionada fue de 80 docentes que representa el 33,3\% de una población de 240 docentes de dos municipios; en Santiago de Cuba se trabajó en el Instituto Preuniversitario Urbano Rafael María de Mendive y en el Instituto Preuniversitario Vocacional de Ciencias Exactas Antonio Maceo Grajales, y en Contramaestre en el Instituto Preuniversitario Urbano Antonio Santiago. Estas instituciones fueron seleccionadas de manera intencional al constituir escuelas representativas por su alta matrícula y relevancia social para la educación preuniversitaria en la provincia Santiago de Cuba.

\section{ANÁLISIS Y DISCUSIÓN DE LOS RESULTADOS}

\subsection{Etnografía del pensamiento pedagógico en relación a las habilidades socioemocionales en el contexto santiaguero.}

En el modelo actuante del docente en Santiago de Cuba se identifican prácticas que etnográficamente permiten conformar una representación del pensamiento profesional pedagógico del docente, el directivo y el profesor guía ${ }^{1}$. En el sistema educativo se entiende por profesor guía aquel docente que por su preparación y

\footnotetext{
${ }^{1}$ Con el objetivo de simplificar el registro etnográfico en lo adelante cuando se emplee un término o frase del modelo percibido, registrada en el trabajo de campo se hará con letra itálica o cursiva. (Nota de los Autores)
} 
experiencia la administración de la escuela le ha asignado la responsabilidad de acompañar y representar el grupo de estudiantes en un rol tutoral que lo convierte funcionalmente en mediador entre los estudiantes y el colectivo de docentes con que trabaja de manera que, coordina actividades, diagnostica y mide impacto del sistema de influencias generado por el claustro con el grupo.

En el modelo percibido actuante de las escuelas estudiadas, a la persona que se gradúa e inicia su actividad profesional como docente se le denomina egresado. Esta denominación homogeneiza y despersonaliza, pues en la composición del colectivo ocupan el componente diferenciador de egresado. Dentro de los significados que tiene para los docentes ser egresado está que al no tener la experiencia de trabajo en ese contexto laboral específico, al estar comenzando su historia profesional laboral y al haber salido de los estudios teóricos de la universidad le falta preparación y requiere de atención diferenciada.

Esta representación presente en el modelo percibido al someterlo a los análisis del modelo operatorio requiere de interpretación y análisis de sus significados pues afectan los procesos de cambios institucionales, la adaptación al medio laboral y el uso de las potencialidades de la liminalidad en el contacto generacional para el perfeccionamiento de la actividad profesional pedagógica.

Dentro del trabajo de campo realizado en la valoración de las clases y de la calidad en la formación de los egresados, se aprecia que docentes técnicamente bien preparados ${ }^{2}$ se sienten limitados por no poseer suficiente experiencia para lograr disciplina, atención y disposición en los estudiantes del grupo con que trabajan. Por regla general, se observa que en la medida en que el nivel de preparación del egresado, en la didáctica y el contenido de la disciplina docente que imparte disminuye, la carencia para lograr los aspectos señalados se agrava. Esta es causa que docentes de experiencia y directivos argumentan, por la cual, en ocasiones, recurren a procedimientos inadecuados como el regaño, la humillación a los estudiantes o la violencia verbal o física. En el modelo percibido se transmite la regla sintetizada en la frase usada por los docentes: en el aula mando yo, de la cual se infiere un estilo autoritario y vertical en las relaciones interpersonales entre docentes y estudiantes.

En el año 2016 y 2017 se registraron incidencias en las que docentes emplearon procedimientos inadecuados en el proceso docente educativo y los estudiantes y familias se sintieron maltratados. Por otra parte, en la observación semi-estructurada

\footnotetext{
${ }^{2}$ Entendiendo por ello, tener dominio de la didáctica y el contenido de la disciplina docente que imparten. (Nota de los Autores)
} 
realizada se pudo apreciar que existe un uso frecuente del regaño y de la disciplina rígida en el desarrollo de actividades docentes en la que el monopolio de la palabra y la transmisión de información caracterizan la comunicación. En docentes de experiencia se observó que emplean un tiempo excesivo durante la clase para realizar reclamos de comportamientos y censuras de conductas inapropiadas que en la cultura escolar son denominadas por los estudiantes y docentes como pelear.

Los directivos, frecuentemente sometidos a la necesidad de mediar en la solución de conflictos, recurren, en casos difíciles, a la expulsión del centro como solución a un estudiante que transgrede con frecuencia las normas de la institución, sin razonar que al trasladar al estudiante lo único que sucede es el traslado de los problemas a otro ámbito institucional de similares características por lo que pedagógicamente no sería realmente una solución del conflicto. La aplicación de esta medida genera un comportamiento frecuente en la amenaza para lograr concientizar en el cumplimiento del reglamento establecido por la institución que hace frecuente el uso por el director de la frase el que no cumpla o el que incurra en indisciplina grave se va de la escuela.

Los profesores guías en el trabajo con la familia, al realizar las reuniones de padres con una frecuencia, por lo general, mensual. En ellas se observa la tendencia de convertirla en un espacio para dar quejas de los estudiantes con problemas de comportamiento o bajo desempeño, comunicar en público las deficiencias detectadas en la realización del proceso educativo. Esto ocurre a pesar de existir la indicación oficial que debe predominar la orientación educativa y el desarrollo coordinado de acciones en la educación de los adolescentes.

En el caso de los estudiantes de alto rendimiento la tendencia es al estímulo en las reuniones de padres con frases como él no tiene problemas, es un buen estudiante. Por lo general, las familias de los estudiantes con más problemas son los que se ausentan a las reuniones de padres. La ejecución de estas reuniones se convierte en una interacción tensa en que la queja, la demanda y el señalamiento en contraposición con el elogio, son tenidos como los procedimientos en los que se sustentan las pautas culturales con las que se valora, en el modelo percibido, su calidad de padres y estudiantes. 
Las descripciones realizadas denotan la existencia de situaciones educativas que requieren de un tratamiento en la forma de representarse los docentes el desarrollo de la labor educativa en la que se requiere incorporar el desarrollo de habilidades socioemocionales en la gestión de aprendizaje en el nivel Medio Superior en Santiago de Cuba.

\subsection{Sistematización conceptual de habilidades socioemocionales en la gestión de aprendizaje}

Las situaciones educativas determinadas descriptivamente en el contexto santiaguero, enfocan la necesidad del desarrollo de habilidades socioemocionales para el perfeccionamiento de la labor pedagógica. Por estas, se entienden comportamientos observables, aprendidos y utilizados en las relaciones sociales para el intercambio de información y la interacción para obtener fines concretos. Son prácticas cognitivas concretas que forman parte de una estrategia más amplia de actuación en la labor educativa.

No hay un acuerdo conceptual ni existe una definición única de habilidad socioemocional, en la sistematización conceptual se partió de los estudios de Secord y Backman, (1976), Blanco, (1981), Caballo, (1986), Kelly, (1992), y Gismero, (2000). En estos trabajos se identificaron características relevantes para el tratamiento a habilidades socioemocionales como: comportamientos manifiestos aprendidos, orientación a objetivos y metas, especificidad contextual situacional y cultural, integración de aspectos conductuales observables y cognitivos y afectivos no observables directamente.

Las habilidades socioemocionales orientan a la búsqueda de la conformación de una inteligencia social entendida esta, en una primera aproximación, como una habilidad para comprender y dirigir a las personas para actuar prudentemente y constructivamente en las relaciones humanas.

Un elemento importante en la caracterización de las habilidades socioemocionales lo tienen los sentimientos. Estos son el componente experiencial subjetivo de la emoción con el cual el individuo evalúa la experiencia tras un evento emocional. Ello hace necesario realizar distinciones entre los sentimientos y las emociones en el estudio de las habilidades socioemocionales.

Los sentimientos son la predisposición de los individuos a responder afectivamente ante cierto tipo de acontecimientos. Es una actitud cognitiva de alcance valorativo que se traducen en motivaciones latentes que caracterizan a una personalidad. Por 
su parte, se considera pertinente el criterio de Mora (2008), que las emociones son actitudes motivadoras, que movilizan al individuo a conseguir o evitar lo que es benéfico o dañino para la persona y tiene una existencia circunstancial o eventual.

Las emociones según Reeve (2004) refieren una existencia como fenómenos subjetivos, biológicos, propositivos y sociales e identifica tres componentes de la emoción con los que se coincide en esta investigación: 1) el componente subjetivo le da a la emoción un sentimiento, una experiencia subjetiva que tiene significado personal. La emoción se siente a un nivel subjetivo, tanto en intensidad como en calidad. 2) El componente biológico incluye la actividad de los sistemas autónomo y hormonal, ya que participan en la emoción para preparar y regular la conducta de enfrentamiento adaptativo. 3) El componente funcional da cuenta de cómo una emoción, una vez experimentada, beneficia al individuo. En este componente se puede hablar de normas culturales socioemocionales establecidas y reconocidas por el grupo social tomando en cuenta las diferentes regiones o territorios en que se desarrolla el grupo.

Estas normas regulan, en una situación concreta, qué debemos sentir, cómo y de qué manera, es decir, la dirección de la emoción, su intensidad y la duración. Cuando nuestra emoción no se corresponde con lo establecido por las normas culturales socioemocionales, se produce una disonancia entre lo que realmente se siente y lo que se debería sentir.

La sensibilidad emocional supone el proceso general de valoración, por el que las emociones pueden ser entendidas como procesos que señalan la relevancia de los estímulos sociales en sus sistemas cognitivos y de acción. La evidencia empírica de las investigaciones en neurociencias realizadas por Damasio, (2001) subrayan la interacción profunda entre la razón, las emociones y la repercusión en el cerebro de todo lo que ocurre en el cuerpo, reafirmando el funcionamiento como un todo de la personalidad.

En la relación sentimientos-emociones se generan en la formación estímulos placenteros o recompensantes que mantienen la supervivencia de comportamientos culturales deseados o no socialmente en la vida social. Un sentimiento propicia la emoción su valoración se produce de manera similar a como se hace durante una emoción. Los sentimientos son disposiciones duraderas que fungen como matrices en la experiencia emocional, pueden llegar a ser manifestadas ante la confrontación real con el estímulo emocional que la activa. 
Del estudio de la relación sentimiento-emoción se enriquece la interacción educativa como lenguaje en que se facilita una comunicación rápida y efectiva. Para el ser humano, el lenguaje emocional es un lenguaje básico que se requiere dominar y enseñar en el proceso de formación pedagógica. La relación afectiva establecida con el lenguaje emocional sirve para almacenar y evocar memorias en el proceso de formación de una manera más efectiva. Un acto educativo asociado a una vivencia emocional, de matiz placentero o de castigo, permite un mayor y mejor almacenamiento y evocación de lo sucedido a sus participantes. Ello tiene claras consecuencias para el éxito en la gestión de aprendizaje en el proceso formativo.

Se ha demostrado en estudios realizados por Fridja (1996) que existen pautas universales para la expresión facial de ciertas emociones básicas como la ira, el enojo, la alegría, el disgusto, la ansiedad y la tristeza, también dejó planteado que la expresión como lenguaje de las emociones puede variar en función de las costumbres, normas y creencias sociales.

La expresión de lo singular y lo universal en la comprensión de las emociones y sentimientos que surge en el manejo de habilidades socioemocionales da cuenta de la existencia de emociones básicas y generales que surgen ante los mismos estímulos y se manifiestan con expresiones o gestos similares. Unido a esto también se ha probado por Haidt (2003) que existen otras emociones dependientes en mayor medida de la cultura, de una determinada dinámica social contextual y de una evaluación previa a su manifestación de alcance moral.

De la relación sentimiento-emoción contextualizado en una relación singular-universal se sistematiza en este estudio el desarrollo de las habilidades socioemocionales que en la década de los años 1990 fue denominada por Salovey y Mayer como inteligencia emocional. Para apuntar que la emoción afecta, no sólo a los contenidos del pensamiento, también a los propios procesos implicados en éste y en las interacciones sociales, aspecto que debe ser tenido en cuenta en la realización de la labor pedagógica de los docentes. Con mayor precisión Brackett y Caruso (2007) refieren que las emociones y las habilidades están relacionadas con su manejo y éstas afectan los procesos de aprendizaje, las relaciones sociales, el rendimiento académico y laboral.

Lo anterior justifica la asunción de una educación emocional en la formación inicial y permanente de los docentes como una forma de prevención primaria de comportamientos no pertinentes a la labor educativa. En esta educación emocional 
se garantiza la adquisición de saberes aplicables a múltiples situaciones como: la prevención del consumo de drogas, prevención del estrés escolar, depresión escolar y laboral y la violencia.

La prevención primaria como cualidad resultante de la educación socioemocional pretende minimizar la vulnerabilidad de la persona a determinados problemas docentes y escolares de repercusión social y a prevenir su ocurrencia. El carácter de prevención primaria tiende a confluir con la intencionalidad en la educación para maximizar las tendencias constructivas y minimizar las desviaciones formativas en ocasiones fruto de la falta de preparación para el tratamiento a las habilidades socioemocionales.

Estas aportaciones son de especial relevancia para el diseño de intervenciones educativas en el diseño del plan de estudios, talleres, programas y seminarios destinados a fomentar el desarrollo de las habilidades socioemocionales. La aplicación práctica en la educación es un tema que requiere de contextualización y estudio en el proceso de gestión de aprendizaje. Teniendo como antecedente las bases epistemológicas sistematizadas por Saarni (2000). Este autor, pone especial acento en la importancia del reforzamiento de la autoconciencia, la regulación emocional, la automotivación y las habilidades socioemocionales, para facilitar una comunicación eficaz y mayor cooperación de los agentes educativos, aspectos necesarios de continuar fomentando en el contexto educativo santiaguero.

Se identificaron tres dimensiones para el desarrollo de habilidades socioemocionales:

- Dimensión conductual se realiza en los diferentes contextos de actuación. Los componentes conductuales de las habilidades socioemocionales son: no verbales (mirada, contacto ocular, sonrisa, gestos, postura corporal); paralingüísticos (cualidades de la voz como el volumen, claridad, timbre, velocidad, tono); y verbales (de contenido como peticiones, consentimiento, alabanzas, preguntas) y conversación en general. Capacidad para elaborar un modelo preciso de sí mismo y utilizarlo apropiadamente para interactuar de forma efectiva en el proceso de formación.

- Dimensión personal abarca las habilidades cognitivas, afectivas y fisiológicas. Se expresa en emociones como la ansiedad, ira o manifestaciones fisiológicas como la presión sanguínea, ritmo cardiaco, relajación. Habilidades para el liderazgo, resolver conflictos, empatía (comprender a los demás, ver qué les motiva, cómo trabajan.) 
- Dimensión situacional concreta en el contexto ambiental y social, refiere a problemas, conflictos, auto-observación, autocontrol, análisis social (observar a los demás para saber cómo relacionarnos con ellos de forma productiva).

Para el desarrollo de habilidades socioemocionales se requiere la conformación de condiciones educativas que a su vez son expresión progresiva de la realización de estas a partir de crear: a) Una actitud positiva en los participantes en el proceso de enseñanza aprendizaje. b) Reconocer los sentimientos propios y las emociones por cada uno de los participantes. c) Capacidad para expresar sentimientos y emociones. d) Capacidad para controlar sentimientos y emociones propios. e) Capacidad empática de ponerse en la situación del otro. f) Capacidad para tomar decisiones adecuadas. g) Motivación, ilusión e interés. h) Adecuada autoestima. i) Capacidad de resiliencia que alude a actitudes para superar las dificultades y frustraciones.

En el punto de vista epistemológico Sacks (1992) destaca tres grandes modelos conceptuales de habilidades sociales cuyo análisis resultó de interés pues sintetizan las tendencias para su estudio: 1) el modelo de los rasgos, 2) el modelo molecular o centrado en los componentes, y 3) el modelo del proceso o de sistemas.

El primero: modelo de los rasgos asume que las habilidades socioemocionales están predeterminadas e incluidas en la estructura de personalidad de los individuos. Este modelo orienta tener en cuenta la cuestión biológica aunque sin absolutizaciones pues los autores se acogen a la concepción de la unidad bio-psico-social del desarrollo de la personalidad.

El segundo: modelo centrado en los componentes en los que las habilidades socioemocionales son unidades observables de comportamientos verbales (preguntas, autoafirmaciones, elogios, peticiones, etc.), no verbales aprendidos (miradas, gestos, sonrisas, orientación, expresión facial, expresión corporal, contacto, distancia-proximidad), cognitivos y emocionales (conocimiento social, capacidad de ponerse en el lugar de otras personas, capacidad de solucionar problemas sociales, constructos personales, expectativas, planes y sistemas de autorregulación) que, combinados, producen interacciones exitosas en situaciones específicas en que las personas utilizan estas habilidades en situaciones interpersonales para obtener o mantener reforzamiento de buenas prácticas en los contextos de actuación. Una gestión de aprendizaje apoyada en una labor educativa centrada en los componentes basa la formación en el reforzamiento de cada parte del comportamiento considerado en la experiencia como pertinente. 
El tercero: modelo centrado en el proceso, las habilidades socioemocionales son componentes de acciones específicas (verbales, no verbales y comportamientos) o secuencias de comportamientos que crean encuentros concretos (como los saludos, el establecimiento de orden en la clase o la interacción comunicativa en la enseñanza aprendizaje de un contenido) que están regulados. Estos componentes se aprenden mediante la experiencia y la observación, se almacenan en la memoria de forma simbólica y son, luego, recuperados para utilizarlos en la construcción de situaciones concretas de actuación social.

Los autores de esta investigación asumen el análisis dialéctico de esta triada de modelos para llamar la atención en la necesidad de construir metas que definan motivaciones para alcanzar comportamientos socialmente pertinentes, basados en el fomento de la habilidad para percibir el contexto de actuación y las necesidades individuales propias y ajenas, concretándose en lo que la persona dice, hace, piensa y siente.

Del análisis dialéctico de los modelos de estudio de habilidades socioemocionales abordados esta investigación identifica como habilidades socioemocionales básicas para la gestión de aprendizaje en el contexto santiaguero las siguientes: a) Habilidad comunicativa para percibir, valorar y expresar emociones con exactitud. b) Habilidad asertiva para acceder, reconocer y generar sentimientos que faciliten el pensamiento crítico, constructivo y motivador de sí mismo y de los demás. c) Habilidad empática para comprender emociones y el conocimiento emocional. d) Habilidad interactiva de autocontrol para regular las emociones promoviendo un crecimiento emocional e intelectual. e) Habilidad decisoria para lograr la solución de problemas interpersonales, laborales y cognitivos a partir del consenso y la participación. f) Habilidad predictiva pro social para asumir riesgos razonables en la modelación y realización de realidades deseadas en el optimismo, la objetividad y el compromiso social.

\subsection{Procedimiento analítico para la implementación de una pedagogía del desarrollo socioemocional en la gestión de aprendizaje}

Se estructura, con la sistematización realizada, un procedimiento pedagógico matricial con el cual se genera una reflexión que se convierte en marco de realización de un pensamiento dialéctico a partir de la revisión crítica de la realidad social y escolar, a partir de dos diadas conceptuales. Por un lado, la relación requisitospremisas y, por otro, la relación de lo social y lo escolar que, se esquematiza y da lugar al siguiente gráfico. 
GRAFICO. 1

Matriz analítica pedagógica para el desarrollo socioemocional (elaboración propia de los autores)

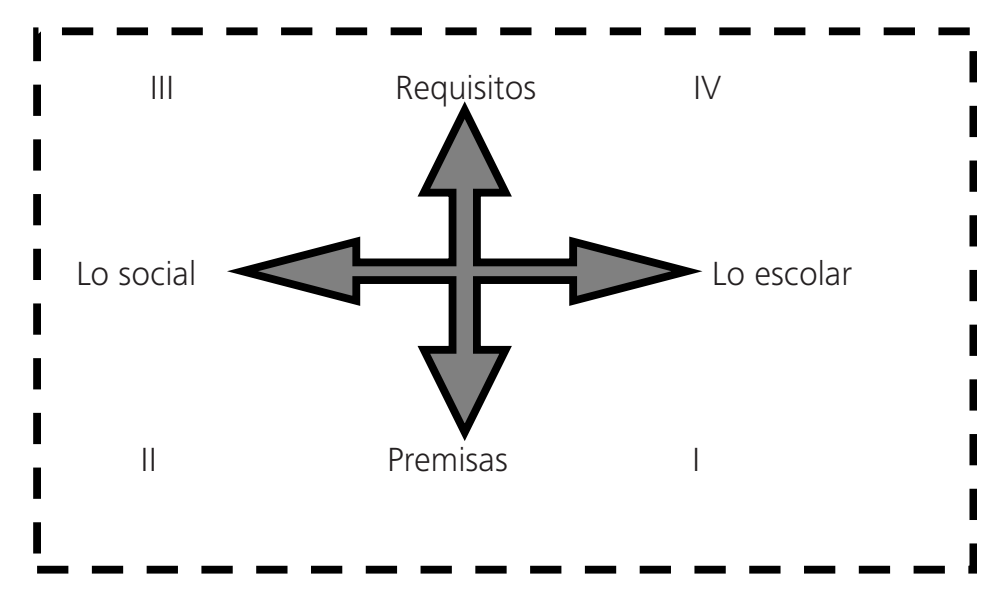

La matriz graficada parte, en este caso, de asumir como campo temático ${ }^{3}$ las habilidades socioemocionales, y estructura una lógica sistémica conformada por dos componentes sintetizados en diadas que en su relación conforma cuatro cuadrantes. Al ser aplicados al análisis de la realidad social brindan información necesaria para el diseño e implementación de una gestión de aprendizaje que favorece la toma de decisiones pedagógicas en el desarrollo de habilidades socioemocionales.

Las premisas son punto de partida y dan cuenta, de forma operacional, de elementos fácticos y fenomenológicos contextuales del estado de la relación y que, en ese nivel, reflejan características del objeto de análisis basado en la información empírica obtenida. Sintetizan el ser cultural al estar conformadas por formas culturales y representaciones sociales establecidas en el modo de actuación de los sujetos implicados. Son normas culturales objetivadas, establecidas como cotidianas, válidas y plausibles.

Los requisitos son ideales que sintetizan el deber ser para la sociedad, aspiraciones que recogen representaciones sociales deseadas, construcciones culturales de existencia situacional, pero de carácter universal, al ser consensos de formas

\footnotetext{
${ }^{3}$ Este concepto de orden operacional ha sido sistematizado por Justis (2013) y por él, se entiende la definición, en ejercicio de abstracción, del espacio de la realidad social que será objeto de análisis en el aplicación del procedimientos analítico pedagógico. (Nota de los Autores)
} 
culturales prevalecientes que orientan la conformación de comportamientos por los individuos bajo las formas culturales y representaciones homogeneizadas para los miembros del grupo.

La diada de lo escolar y lo social establece otras relaciones de carácter dialéctico conformando otro componente del sistema de relaciones que se construye con el procedimiento pedagógico matricial. Delimita dos contextos culturales generales que se conforman como estructuras estables contentivas de relaciones esenciales para la comprensión de la realidad social concreta en que se desarrollan las habilidades socioemocionales a partir de la implementación de la matriz conformada de las relaciones dialécticas derivadas de las diadas.

Se entiende por matriz la estructuración lógica que en su dialéctica concretada en diadas, en el punto de vista conceptual, permite establecer relaciones generadas en una estructuración didáctica en filas y columnas que delimitan cuadrantes de análisis que luego de conformados actúan con otros que se generen en el marco categorial de las ciencias sociales, de la educación y pedagógicas.

\begin{tabular}{|l|l|l|}
\hline & \multicolumn{1}{|c|}{ Lo escolar } & \multicolumn{1}{c|}{ Lo social } \\
\hline & $\begin{array}{l}\text { Estudio diagnóstico institucional de la } \\
\text { práctica de habilidades sociales. } \\
\text { Estudio diagnóstico institucional de uso } \\
\text { (ser cultural) } \\
\text { y fomento de emociones y sentimientos. } \\
\text { Elaboración de proyectos institucionales } \\
\text { para la gestión de aprendizaje. }\end{array}$ & $\begin{array}{l}\text { Estudio etnográfico sobre habilidades sociales } \\
\text { en actividades sociales de convivencia social } \\
\text { (transporte, alimentación, disciplina social, } \\
\text { violencia social, higiene). } \\
\text { Elaboración de situaciones sociales a partir } \\
\text { de vivencias personales para conformar casos } \\
\text { de estudio y desarrollar análisis reflexivo en el } \\
\text { marco de las diferentes instituciones. }\end{array}$ \\
\hline $\begin{array}{l}\text { Requisitos } \\
\text { (deber ser) }\end{array}$ & $\begin{array}{l}\text { Elaboración de objetivos institucionales } \\
\text { para el desarrollo de habilidades } \\
\text { socioemocionales en la gestión de } \\
\text { aprendizaje. } \\
\text { Modelación institucional de } \\
\text { significados, comportamientos del } \\
\text { ciudadano que la sociedad necesita } \\
\text { para su desarrollo sostenible. }\end{array}$ & $\begin{array}{l}\text { Identificación de problemas sociales que } \\
\text { afectan la convivencia social. } \\
\text { Identificación de las potencialidades sociales } \\
\text { que caracterizan al territorio. } \\
\text { Identificación del ideal grupal de ser humano } \\
\text { al que aspira el grupo social. }\end{array}$ \\
\hline
\end{tabular}

Estas determinaciones se convierten en contenidos que se organizan en actividades, acciones y tareas del proyecto educativo que se crea con las instituciones implicadas en cada comunidad. Son el resultante del análisis, en cada cuadrante, de la matriz del comportamiento de elementos culturales dados en premisas o requisitos y que impactan en la práctica social como debilidades, fortalezas, estrategias, acciones y actividades con las que se concibe la formación. 
El carácter pedagógico de la matriz está dado por ser vía para concretar el compromiso social de la institución en el servicio social que presta y que se estructura orientada a la intención educativa en la interacción de factores escolares y sociales para la formación del modelo de persona que cada sociedad pretende alcanzar. Por tanto, su cualidad resultante se convierte en una propuesta concreta a cada institución para mejorar la práctica educativa en la red social educativa.

El carácter analítico se determina por la contextualización a la cultura específica del grupo que se pretende formar y a la identificación en ella de las premisas y requisitos que limitan y/o favorecen el desarrollo socioemocional en la gestión de aprendizaje a partir de condiciones concretas existentes en el medio social, cultural y tecnológico.

\section{CONCLUSIONES}

Queda demostrada la utilidad del procedimiento pedagógico matricial en la experiencia de este trabajo, que se desarrolla mediado por la matriz analítica pedagógica para el desarrollo socioemocional en el proceso de gestión de aprendizaje. Con la matriz se generan nuevos contenidos contextualizados a la actividad pedagógica de las instituciones, agentes e instancias con responsabilidad social con la educación de las actuales y futuras generaciones.

La estructuración de un proceso de enseñanza aprendizaje que tiene por centro el estudiante necesariamente debe profundizar no solo en las habilidades cognitivas también debe enfatizar en el desarrollo socioemocional. Ello hace necesario profesionalizar a los docentes en cómo educar sentimientos, emociones y conocimientos a partir de la cultura propia del grupo social al que pertenece.

\section{BIBLIOGRAFÍA}

Becher, T. (2001). Tribusi y territorios académicos. La indagación intelectual y las culturas de las disciplinas. Barcelona: Gedisa.

Bentaouet-Kattan, R. y Székely, M. (2013). Deserción en la Educación Media Superior de México: patrones, consecuencias y posibles causas. Centro de Estudios Educativos y Sociales, World Bank, Washington.

Bisquerra, R. y Pérez, N. (2007). Las competencias emocionales. Revista Educación XXI. 10 (61-82) España: UNED. 
Bisquerra, R. (2003). Educación emocional y competencias básicas para la vida. Revista de Investigación Educativa (RIE), 21(1), pp. 7-43.

Bisquerra, R. (2009). Psicopedagogía de las emociones. Madrid: Síntesis.

Brackett, M. A. y Caruso, D. R. (2007). Emotionally literacy for educators. Cary. NC: SEL-media.

Blanco, A. (1981). Evaluación de habilidades sociales. En R. Fernández--Ballesteros, y J. A. Carrobles (Eds.), Evaluación conductual: Metodología y evaluación (pp. 568). Madrid: Pirámide.

Caballo, V. (1986). Evaluación de las habilidades sociales. En R. Fernández--Ballesteros, y J. A. Carrobles (Eds.), Evaluación conductual: Metodológica y aplicaciones. Madrid: Pirámide.

Caballo, V. y Buela, U. (1988). Molar/molecular assessment in an analogue situation: Relationships among several measures and validation of a behavioral assessment instrument. Perceptual and Motor Skills, 67, 591- 602.

Damasio, A. (2001). La sensación de lo que ocurre: cuerpo y emoción en la construcción de la conciencia. Madrid: Debate.

De Pedro, F. E (2005). Educar para la resiliencia. Un cambio de mirada en la prevención de situaciones de riesgo social. Revista Complutense de Educación Universidad de Alcalá de Henares, 16 (1), pp. 107-124

Delors, J. (1997). La educación encierra un tesoro. Informe a la UNESCO de la Comisión Internacional sobre la educación para el siglo XXI. México: Dower. Ediciones UNESCO.

Eisenberg, N. y Strayer, J. (1992). La empatía considerada en el contexto. En N. Eisenberg y J. Strayer (Eds.), La empatía y su desarrollo. pp. 425-434. Bilbao: Desclée de Brouwer.

Elizondo, A. Coord. (2001). La nueva escuela, I. Dirección, liderazgo y gestión escolar. México: Paidós.

Extremera, N. y Fernández, P. (2002). El papel de la inteligencia emocional en el alumnado: evidencias empíricas. En Revista Electrónica de investigación Educativa, 6:2. Disponible en http://redie.uabc.mx/vol6no2/contenido-extremera.html [consultado el 02 de julio de 2014].

Extremera, N. y Fernández-Berrocal, P. (2002). Educando emociones. Educación de la inteligencia emocional en la escuela y en la familia. En Fernandez-Berrocal, P. \& Ramos D. N. Corazones inteligentes. España: Kairós.

Fernández, P. y Extrema, N. (2005). La Inteligencia Emocional y la educación de las emociones desde el Modelo de Mayer y Salovery. En Revista Interuniversitaria de Formación del Profesorado. 19(3), pp. 63-94.

Fridja, N. (1996). Passions: Emotion and socially consequential behavior. En: Kavanaugh R, Zimmerberg B, Fein S (eds). Emotion: Interdisciplinary Perspectives, pp. 1-28, Nueva Jersey: Lawrence Erlbaum Associates. 
Gan, F. y Triginé, J. (2012). Análisis de problemas y toma de decisiones. Colección: Monografías. Serie: Administración/Marketing. En Manual de instrumentos de gestión y desarrollo de las personas en las organizaciones. Madrid: Ediciones Díaz de Santos.

Garcia A. D. (2010). Estudio sobre la asertividad y las habilidades sociales en el alumnado de Educación Social. Universidad de Huelva. Revista educación Siglo XXI. pp. 225240. Recuperado de http://rabida.uhu.es/dspace/bitstream/handle/10272/5352/ b16450188. pdf?sequence $=2$

Gardner, H. (1995). Multiple Intelligences as a Catalyst. English Journal, pp. 16-18.

Goleman, D. (1999). La práctica de la inteligencia emocional. Editorial Kairós.

González, R., Montoya, I., Casullo, M. y Bernabéu, J. (2002). Relación entre estilos y estrategias de afrontamiento y bienestar psicológico en adolescentes. Psicothema, 14(2), pp. 363-368.

Gismero, E. (2000). EHS: Escala de Habilidades Sociales. Manual. Madrid: TEA.

Gismero, E. (2001). Evaluación del autoconcepto, la satisfacción con el propio cuerpo y las habilidades sociales en la anorexia y bulimia nerviosa. Clínica y Salud, 12(3), 289-304.

Haidt, J. (2003). The moral emotions. En: Davidson RJ, Scherer K, Goldsmith H (eds). Handbook of Affective Sciences, pp. 852-870. Oxford: University Press.

Justis, O. (2013). La cultura escolar en la toma de decisiones del grupo de docentes en el proceso pedagógico del preuniversitario. Tesis presentada en opción al grado científico de Doctor en Ciencias Pedagógicas. Santiago de Cuba.

Kelly, J. A. (1992). Entrenamiento en habilidades sociales. Bilbao: DDB.

León, J., Jarana, L., y Blanco, A. (1991). Entrenamiento en habilidades sociales al personal de enfermería. efectos sobre la comunicación con el usuario de los servicios de salud. Clínica y Salud, 2(1), 73-79.

López, P. N. y Salovey, P. (2004). Emocional intelligence and social interaction. Personality and Social Psychology Bulletin, 30 (8), pp. 108-134.

Marina, J. A. (2004). Precisiones sobre la Educación Emocional. En Revista Interuniversitaria de Formación del Profesorado. 19(3), pp. 27-44 España.

Mayer, J.D., y Salovey, P. (1997). What is emotional Intelligence? En Salovey, P. \& Sluyter (Eds.) Emotional development and emotional Intelligence: Educational implications, pp. 3-31. Nueva York: Basic books.

Mora, F. (2008). El reloj de la sabiduría. Tiempos y espacios del cerebro humano. Madrid: Alianza

Palomera, R., Fernández, P. y Brackett, M.A. (2008).La inteligencia emocional como una competencia básica en la formación inicial de los docentes: algunas evidencias. España: EOS.

Reeve, J. (2004). Motivación y emoción. México: McGraw Hill 
Repetto, E. (Dir.) (2009). Formación en competencias socioemocionales. Libro del formador. Madrid: La Muralla.

Saarni, C. (2000). Emotional competence. A. Developmental Perspective. In Bar-On. R. y J. D. A. Parker (Eds.) The Handbook of Emotional Intelligence. Theory, Development, Assessment, and Application at Home, School, and in the Workplac. San Francisco, Ca: Jossey-Bass, pp. 68-91.

Sacks, Z. (1992). Habilidades sociales. Recuperado el 14 de Junio del 2015 de http:// www. educacion.once.es/appdocumentos/.../Habilidades-Sociales\%202005.pdf

Sanz, M. L., Sanz, M. T. y Iriarte, M. D. (2000). Reflexiones sobre la enseñanza de las habilidades sociales. Revista de Ciencias de la Educación, 182, 203-216.

Salovey, P. y Mayer, J. D. (1990). Emotional intelligence. Imagination, cognition, and personality, 9, pp. 185-219.

Secord, P. F. y Backman, C. W. (1976). Psicología social. México: McGrawHill. 
